Meeting Report

\title{
Microbial stress: From molecules to systems (Sitges, November 2015)
}

\author{
Francesca Martani ${ }^{1}$, Nadia Maria Berterame ${ }^{1}$, Paola Branduardi* \\ University of Milano-Bicocca, Department of Biotechnology and Biosciences, Milano, Italy
}

\section{A R T I C L E I N F O}

Article history:

Received 21 July 2016

Received in revised form 30 October 2016

Accepted 16 November 2016

Available online 25 November 2016

\begin{abstract}
A B S T R A C T
The meeting "Microbial Stress: from Molecules to Systems" - the third in this series - was held in Sitges (Spain) in November 2015. The meeting offered the opportunity for international scientists to share their viewpoints and recent outcomes concerning microbial stress responses. Particular attention was given to the characterisation of mechanisms triggered by stress, from detailed molecular biology through whole organism systems biology up to the level of populations. A deeper understanding of microbial responses to stress is indeed attainable only considering the phenomenon as a whole. Exhaustive knowledge of the various stress response systems, and of their interconnections, is important for different applications, from the prevention and counteraction of bacterial infectious diseases to the engineering of robust cell factories. The presentations covered all of these aspects, enabling an active interaction among participants. It also stimulated discussions and cross-fertilisation among disciplines, which was one of the aims of the meeting. Moreover, since many stress response mechanisms are broadly conserved, data obtained at the microbial scale may facilitate the comprehension of complex phenomena, such as aging, evolution of neurological diseases and cancer.
\end{abstract}

(c) 2016 Published by Elsevier B.V.
The conference was chaired by Michael Sauer (BOKU University, Natural Resources and Life Sciences Vienna, Austria), with the cochairing of Paola Branduardi (University of Milano-Bicocca, Italy) and Peter Lund (University of Birmingham, UK), on behalf of the Microbial Physiology Section of the European Federation of Biotechnology.

Almost 90 delegates, both from Academia and from Industry, attended the conference with representatives from Europe, USA, Japan, Korea, China, Brazil and Pakistan.

\section{Cross-fertilisation of ideas and techniques to unearth microbial stress response}

The programme was composed of three sessions concerning different aspects of the microbial stress response, considered as a key element for ( $i$ ) the adaptation to intra- and extra-cellular

\footnotetext{
* Corresponding author at: Piazza della Scienza 2, 20126, Milano, Italy. E-mail addresses: francesca.martani@unimib.it (F. Martani), n.berterame1@campus.unimib.it (N.M. Berterame), paola.branduardi@unimib.it (P. Branduardi).

1 These authors contributed equally to this work.
}

environmental changes, (ii) cell performance in industrial production processes and (iii) cross talk with higher organisms. A key feature of the meeting was to emphasise the relevance of the microbial physiology in relation to the bioprocessing industry.

Among the most relevant factors that impair microbial metabolism, and consequently the ability to produce compounds or proteins of interest efficiently, are the stressing conditions imposed by industrial bioprocesses, often related to the substrates used. Presentations during the second session illustrated the effects of such stressors on microorganism physiology, as well as different strategies to improve cellular properties in order to increase the production and the yield of fermentations. These enhancements can only be achieved through an in-depth knowledge of the mechanisms that steer the microbial stress response. The investigation of reactions and networks evolved by microorganisms to deal with stresses encountered in natural or artificial habitats, explored in the first and third sessions, are therefore essential. Beyond doubt, cross-fertilisation of ideas and techniques is indispensable to gain a greater understanding of microbial response to stress (Fig. 1). Accordingly, the division of the programme into three research themes covering broad aspects of stress responses, rather than focusing on single stresses or 


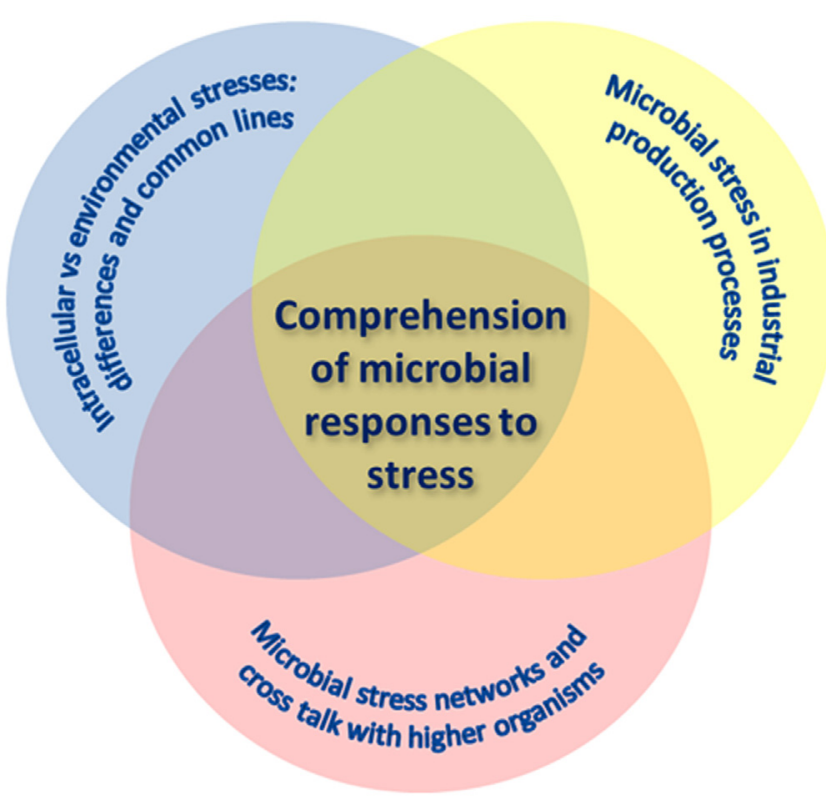

Fig. 1. Cross-fertilisation among disciplines as an indispensable approach for a deeper understanding of microbial stress response.

organisms, promoted stimulating discussion and an unbiased point of view and comments from scientists not necessarily belonging to the same field of research.

\section{Intracellular vs. environmental stresses: differences and common lines}

The survival of microorganisms under stressful conditions relies on regulatory networks that enable the sensing of environmental and/or intracellular changes and the activation of an appropriate response. This complex regulation relies on the interaction of different molecules of all types, including DNA, RNA, proteins, lipids and metabolites. The presentations of this session showed different (and complementary) mechanisms exploited by microbial cells to cope with extracellular as well as intracellular stress, providing many examples of the complexity of stress responses.

Hiroshi Takagi (Nara Institute of Science and Technology, Japan) intriguingly described how the nitric oxide (NO)-dependent antioxidative system is mediated in baker's yeast by the activation of the copper-sensing transcription factor Mac1 [1]. Microarray and real-time quantitative PCR analyses revealed that copper metabolism-related genes regulated by Mac1 are induced by exogenous NO treatment; accordingly, NO synthesised under high-temperature stress increases the level of the CTR1 (high-affinity $\mathrm{Cu}^{2+}$ transporter 1) transcript, intracellular copper content, copperdependent Sod1 (superoxide dismutase 1) activity, and the consequent cell viability. Interestingly, yeasts engineered to over-produce NO showed improved fermentation abilities when exposed to air-drying, high sugar concentrations and freeze-thaw stresses. When present at low concentrations, NO acts as a signaling molecule preserving cells from death, but high concentrations of NO are toxic. The sensing of NO levels, as well as the activation of mechanisms to counteract its toxicity, are therefore crucial in determining the triggering of the stress response.

Jeff Cole (University of Birmingham, UK) presented a coordinated system based on proteins YtfE and $\mathrm{Hcp}-\mathrm{Hcr}$ for the prevention and repair of nitrosative damage during anaerobic growth, a physiologically relevant condition for pathogenic gastrointestinal bacteria [2]. He demonstrated that the hybrid cluster protein, Hcp, is a high affinity NO reductase that is inactivated by its toxic substrate unless protected by its partner protein, Hcr.

Decreased cytosolic $\mathrm{pH}$, generally recognised as stress, was shown by Gertien Smits (University of Amsterdam, Netherlands) to be a signal through which cells experience the environment [3]. It determines whether energy is used for growth or for stress resistance. Indeed, she showed that yeast mutants with defective cell wall integrity or deficient in $\mathrm{Ca}^{2+}$ sensing were sensitive to $\mathrm{pH}$ 2.5 , but were able to survive at $\mathrm{pH} 1.5$, thanks to the intracellular $\mathrm{pH}$ decrease occurring only at extremely low external $\mathrm{pH}$. Cell survival at low $\mathrm{pH}$ was also illustrated by Daniela De Biase (Sapienza University of Rome, Italy) who found a connection between the more acid-resistant phenotype of a new Brucella species and the presence of a functional GAD (glutamate decarboxylase) system, which enables these pathogens to adapt in certain natural habitats and/or in the gastrointestinal tract of their hosts [4].

Metal availability represents another critical factor in determining the outcome of host-pathogen interactions. John D. Helmann (Cornell University, USA) described a Bacillus subtilis model for the maintenance of zinc $\left(\mathrm{Zn}^{2+}\right)$ homeostasis, which is regulated by the metalloregulatory proteins Zur and CzrA that are sensitive to $\mathrm{Zn}$ deficiency and excess, respectively. He showed that Zur controls ribosomal proteins that do not contain a zinc binding motif, but which might function to maintain the proper assembly of the ribosome during zinc limitation [5]. Understanding the mechanisms involved in this kind of regulation could be critical for the development of new antimicrobial compounds that alter metal ion homeostasis. In contrast to $\mathrm{Zn}$, silver $(\mathrm{Ag})$ is a metal with no biological functions and is toxic to microorganisms even at low concentrations. Petek Cakar (Istanbul Technical University, Turkey) showed the successful application of evolutionary engineering for the achievement of the up to $250 \mu \mathrm{M} \mathrm{AgNO}$-resistant Saccharomyces cerevisiae strains, resulting from the upregulation of 739 genes and the downregulation of 853 others (unpublished data). Metal response mechanisms provided examples of cross-talk among different stress response pathways. Another example of the high complexity of cellular functions was provided by Kyung-Tae Lee (Yonsei University, Korea), who described the multiple roles of the cytoskeleton-associated protein glycine-rich Cgp1 in regulating growth, stress response, differentiation and pathogenicity of Cryptococcus neoformans (unpublished data).

The production of specific metabolites represents another efficient strategy adopted by some microorganisms to counteract cellular stress. As an example, trehalose is an osmolyte produced as a stress-responsive molecule by several organisms in response to environmental stresses such as heat, cold, desiccation and osmotic stress. Ewa Laskowska (University of Gdansk, Poland) reported that aggregated proteins are removed by trehalose in Escherichia coli cells during recovery from stationary phase and after heat shock. In addition, otsA mutants, defective in trehalose synthesis, displayed various markers of oxidative stress and were unable to form biofilm [6]. Stanislav Obruča (Brno University of technology, Czech Republic) showed the cryoprotectant function of another chemical chaperone, 3-hydroxybutyrate (3HB), in Cupriavidus necator H16 [7]. 3HB monomers form poly-hydroxybutyrate (PHB), a storage material produced by different bacteria. This defence mechanism might therefore be exploited for industrial applications for biomaterial production.

Bacterial cell envelope rearrangement represents the first line of cellular defence against stress. Changes in the fatty acid profile of the cellular membrane of Rhodococcus erythropolis cells exposed to various stresses were reported by Carla de Carvalho (University of Lisbon, Portugal). In particular, under nutrient limiting conditions 
and desiccation, $R$. erythropolis produces specialised lipids, such as triacylglycerols and wax esters [8]. As in the case of PHB described by Obruča, these compounds accumulated under stress condition may have potential industrial applications, for example in biodiesel production.

\section{Microbial stress in industrial production processes}

One of the major obstacles for the development of competitive industrial bioprocesses is the reduced performance of microorganisms due to stress encountered during fermentation. Stress may occur because of the presence of inhibitors, often deriving from the substrate used for media formulation, or from unfavourable growth conditions. Frequently, the product itself becomes toxic when accumulated at high concentration. Among the undesirable effects caused by stress at both the molecular and physiological levels, the decrease in genetic stability of industrial strains may decrease production, productivity and process yields. Wanda Dischert (METabolic EXplorer, France, http://www.metabolic-explorer.com) presented different strategies adopted by METEX to delay the pressure on bacteria, including the development of a stable genetically engineered $E$. coli strain for the production of glycolic acid, a monomer for biodegradable plastics. Among different approaches, glycolic acid yields can be increased by reducing the expression of the icd (isocitrate dehydrogenase) gene; however, strains with an attenuated expression of this gene were unstable when grown for many generations, a strong disadvantage for industrial use. Strain stability was therefore achieved by METEX through the modulation of icd expression by replacing the natural promoter of this gene with a thermoinducible promoter. Consequently, the optimised process consists of a first phase at $37^{\circ} \mathrm{C}$, where icd is expressed to allow biomass production, followed by a second step at $30^{\circ} \mathrm{C}$, where icd is repressed and glyconic acid is produced.

In the natural environment, but also in large scale industrial cultivation, organisms are exposed to rapid dynamic conditions, which are repetitive. Relevant cultivation parameters, such as dissolved oxygen, substrate concentration, $\mathrm{pH}$ and temperature do vary in largescale bioreactors because of long mixing times. Thus, microorganisms in the bioreactor experience rapid environmental changes that may affect performance. How to study these fluctuations is not trivial. Aljoscha Wahl (Delft University of Technology, Netherlands) presented an experimental approach based on a dynamic shift between feast and famine regimes (400 s and $1800 \mathrm{~s}$ ) that led to repetitive cycles with moderate changes in substrate availability in an aerobic glucose cultivation of $S$. cerevisiae [9]. After a few cycles, the feast/famine switches produced a stable and repetitive pattern with a reproducible metabolic response time, thus providing a robust platform for studying the microorganism's physiology under dynamic conditions. He showed that the biomass yield was slightly decreased (by $-5 \%$ to $-25 \%$ ) under the feast/famine regime, while the averaged substrate and oxygen consumption as well as the carbon dioxide production rates were comparable. Comparing the short and long cycles, he showed that despite a 10-fold change in extracellular glucose concentration, the intracellular concentration and fluxes of glycolytic intermediates only responded with a change of 1.2-1.5-fold. In contrast, intermediates of carbohydrate storage metabolism, such as trehalose 6-phosphate (T6P), reacted more dramatically. Therefore, these results confirmed the adaptability of cellular metabolism and show that storage carbohydrates are key metabolites for yeast robustness.

Another limitation due to large-scale mixing that can be related to fluctuations is that the cells are repeatedly exposed to zones with varying substrate concentrations. Thus, heterogeneities in large reactors might impose stress on the microbial hosts resulting in performance losses. In particular, Johana Simen (University of Tuebingen, Germany) described the impact of large-scale carbon and nitrogen gradients on E. coli K-12 W3110 [10]. Short-term dynamics were found not only for intracellular pools of related nucleotides and alarmones, but also for mRNA levels. In all, about 300 genes were found to be differentially expressed after a residence time of $110 \mathrm{~s}$ in the starvation zone. Analysis of long-term adaptation revealed cellular strategies to cope with the imposed oscillating stress conditions.

However, coping with stress is something that cells experience in natural environments as well, offering the possibility to study how to turn this ability into an industrial advantage. Joseph Shiloach (National Institute of Diabetes Digestive and Kidney Disease/NIH, USA) reported an example of how oxidative stress faced by cells during growth can be exploited for the production of heterologous proteins. He showed that in response to high oxygen concentrations, soxS expression increased 16-fold in E. coli, making its promoter a possible candidate for inducing recombinant protein expression under these conditions [11]. Induction of recombinant protein expression by increasing the dissolved oxygen concentration was found to be a simple and efficient alternative expression strategy that excludes the use of chemical, nutrient or thermal inducers that have a potential negative effect on cell growth or on product recovery.

With a similar perspective, Bettina Loràntfy (Chalmers University of Technology, Sweden) illustrated how the presence of oxygen can be advantageous and in particular can improve acidification capacity of some lactic acid bacteria (LAB). LAB have been used in cheese making for thousands of years by exploiting their capability for the anaerobic production of acidic by-products derived from the fermentation of milk sugar. A recent discovery has shown that some LAB, such as Lactococcus lactis, are able to sustain respiration under aerobic conditions when hemin is added to the growth medium, since it completes the electron transport chain, which is otherwise defective. She showed that, although oxygen represents an additional stress for $\mathrm{LAB}$, in the presence of hemin under aerobic conditions they performed surprisingly better in terms of higher lactate yield (unpublished data).

As mentioned above, stressors that negatively affect production parameters are often derived from the substrate used for media formulation. This is one of the challenges to tackle for the viability of a bio-based economy, where fuels, chemicals and materials are supposed to be produced from lignocellulosic biomass. These substrates are often problematic, both because of their heterogeneous nature and their complexity. In addition, the necessary pre-treatments of lignocellulosic biomasses release inhibitory compounds, in the form of furans, weak acids and phenolic derivatives, which impair cell growth and, in consequence, the yield and production of the process. Strategies to improve cellular robustness may generally comprise decreased uptake or increased efflux of inhibitory compounds, increased detoxification reactions and ability to repair the damage caused by stressful conditions.

Lisbeth Olsson (Chalmers University of Technology, Sweden) presented a toolbox for obtaining cellular robustness in the yeast $S$. cerevisiae with the goal of increasing cell performance. In particular, three examples in lignocellulose-derived streams were described. The first involved a cellular rewiring: Olsson showed that increasing the intracellular levels of glutathione (recognised as scavenger for free radicals and a metabolite relevant for the maintenance of the redox potential) correlates with an enhanced ethanol production of $70 \%$ compared to the control strain [12]. The second example reported was the correlation between increased conversion of phenolic compounds and the resulting increased robustness. In particular, a recent publication showed that the expression of coniferyl aldehyde dehydrogenase (CALDH) from 
Pseudomonas increases endogenous conversion of coniferyl aldehyde into the less toxic ferulate, and that the endogenous acetaldehyde dehydrogenase (Ald5) is actively involved in the catabolism of the phenolic compound in S. cerevisiae [13]. In the latter example, attention was focused on acetic acid, one of the most abundant toxic compounds released during lignocellulose pre-treatments, and its effect on the plasma membrane. This cellular structure plays a crucial role as a flexible but effective element in the cellular response to stress. Studies on Zygosaccharomyces bailii and $S$. cerevisiae cell membranes highlighted how sphingolipid content affects acetic acid permeability, making membrane engineering a possible approach to increasing cellular robustness toward inhibitory compounds [14].

Weak acid tolerance was also investigated by Nurzhan Kuanyshev (University of Milano-Bicocca, Italy) who focused attention on the industrially relevant yeast Kluyveromyces marxianus. Genome analysis revealed the presence of additional copies of the genes PDR12 and PDR5 compared to $S$. cerevisiae (unpublished data): these genes encode weak acid transporters in baker's yeast and the gene amplification suggests a different evolution that occurred in K. marxianus. A specific transcriptomic analysis showed a peculiar induction profile of these transporters that was extended from exposure to acetic to other organic acids. These results highlight once more the important role of the cell membrane in organic acid tolerance/intracellular concentration, as previously presented by Lisbeth Olsson.

Marco Brambilla (University of Milano-Bicocca, Italy) gave another example of cellular rewiring connected to strain robustness. He showed that the alteration of a post-transcriptional master regulator element might represent a novel approach to unlock industrially promising phenotypes. The modulation of the levels of the poly(A) binding protein Pab1 (a stress granules component) increased $S$. cerevisiae robustness against acetic acid, $\mathrm{H}_{2} \mathrm{O}_{2}$ and heat stress. In addition, mutagenesis of $P A B 1$ and the application of a specific screening protocol on acetic acid enriched medium allowed the isolation of the further ameliorated mutant pab1 A60-9, characterised by defects in mRNA deadenylation [15].

In addition to the toxicity of the compounds released from the treatment of biomass, one of the major limitations for a viable production is the toxic effect of the very high concentrations of product reached during the fermentative process. Nuno Pereira Mira (University of Lisbon, Portugal) presented an experimental system to identify, at a genome-wide scale, the genes and pathways required for maximal tolerance of $S$. cerevisiae to the stress induced by an important building block molecule, itaconic acid. He showed an enrichment of genes involved in different pathways, including the identification of a plasma membrane transporter that serves as an itaconic acid/itaconate exporter (unpublished data).

Whatever the nature of the stress, the use of biosensors offers possibilities for improving cells for the synthesis of molecules of interest. Jan Dines Knudsen (Lund University, Sweden) described an efficient tool to measure the impact of environmental perturbations or heterologous gene expression on cellular NADH levels [16]. The biosensor is based on the expression of green fluorescent protein (GFP) under the control of the glycerol-3phospate dehydrogenase (GPD) 2 promoter, responding to changes in the NADH/NAD ${ }^{+}$ratio. The reporter system displayed high resolution in distinguishing cytosolic NADH oxidation capacity and hence has potential to be used in high-throughput screening for selecting improved variants based on single cell fluorescence.

\section{Microbial stress networks and cross-talk with higher organisms}

Regulatory networks allow complex physiological responses mediated by coordinated changes at both the molecular and cellular levels, enabling pathogens to cope with the stressful conditions imposed by the host organism. The sigma factor SigE of Mycobacterium smegmatis, together with its anti-sigma factor RseA and the MprA/MprB two-component system, was described by Fedora Babić (University of Padova, Italy) as a dynamic network whose perturbation affects bacterial survival under various stress conditions (unpublished data). Grzegorz Wegrzyn (University of Gdansk, Poland) showed that oxidative stress, usually occurring in the human intestine, induces the Shiga toxin-converting prophage in enterohaemorrhagic $E$. coli strains through the OxyR protein [17]. Prophage induction was also evoked by human stress hormones; on the other hand, starvation stress inhibited Shiga toxin production, suggesting that this mechanism is tightly regulated by the cellular response to environmental conditions. The influence of host-imposed stresses on microbial response was also highlighted by Alistair J. P. Brown (University of Aberdeen, United Kingdom), who illustrated the significance of combinatorial cationic plus oxidative stresses in host defences against Candida albicans. He proposed a mechanism in which cations interfere with the intracellular response to $\mathrm{H}_{2} \mathrm{O}_{2}$ exposure, leading to ROS accumulation and killing of the pathogens by neutrophils [18].

Roy Parker (University of Colorado Boulder, USA) illustrated eukaryotic stress granules (SG) as heterogeneous and dynamic mRNA-protein complexes acting in the control of translation and mRNA degradation when translation is inhibited. Super-resolution microscopy reveals that SG contain stable core substructures surrounded by a phase-separated shell [19]. Proteomic analysis of SG cores indicated that the assembly of these granules occurs through a dense network of protein-protein interactions, in which different ATP-driven machines are required. Interestingly, neuronal RNA granules, involved in synaptic plasticity, habituation and memory, share several common features with SG and various neurodegenerative diseases are characterised by pathological granules, which may be linked to defects in ATP hydrolysis. Therefore, mechanisms that are revealed and generally are investigated under stressful conditions may also have a crucial role in physiological functions.

The complexity of biological responses was also shown by Hitoshi Nakamoto (Saitama University, Japan): opposite modulations of a single protein may unexpectedly determine the same effect. He focused attention on the ATP dependent molecular chaperone Hsp90, whose activity is essential for cancer cell survival as many cancer-related proteins are substrates for Hsp90. His research highlighted that, in addition to inactivation, the hyperactivation of the Hsp90 ATPase activity is also harmful to cancer cells [20]. These outcomes are promising for the development of novel therapeutic strategies in killing cancer cells.

In conclusion, the variety of the topics presented covering the investigation of different microorganisms, stresses and applications, as well as the assorted scientific backgrounds of the participants, fostered not only a great exchange of ideas and perspectives, but also a real interaction between academia and industry. The conference encouraged the creation of new contacts between universities, research institutes and industry. The interdisciplinarity that characterises the topic of microbial stress response certainly also characterised the conference. We are already looking forward to the fourth conference in this series, which is planned to be held in about two years from now. 


\section{Acknowledgements}

We thank Michael Sauer and Jeff Cole for critically reading the manuscript and providing helpful comments. Grants for attendance by students and young scientists were sponsored by FEMS. EPPENDORF is also gratefully acknowledged for providing sponsorship including cash prizes for the best posters by young scientists.

\section{References}

[1] Nasuno R, Aitoku M, Manago Y, Nishimura A, Sasano Y, Takagi H. Nitric oxidemediated antioxidative mechanism in yeast through the activation of the transcription factor Mac1. PLoS One 2014;9:e113788.

[2] Cole JA. Legless pathogens: how bacterial physiology provides the key to understanding pathogenicity. Microbiology 2012;158:1402-13.

[3] Orij R, Urbanus ML, Vizeacoumar FJ, Giaever G, Boone C, Nislow C, et al. Genome-wide analysis of intracellular $\mathrm{pH}$ reveals quantitative control of cell division rate by $\mathrm{pH}(\mathrm{c})$ in Saccharomyces cerevisiae. Genome Biol 2012;13:R80.

[4] Damiano MA, Bastianelli D, Al Dahouk S, Köhler S, Cloeckaert A, De Biase D, et al. Glutamate decarboxylase-dependent acid resistance in Brucella spp. distribution and contribution to fitness under extremely acidic conditions. Appl Environ Microbiol 2015;81:578-86.

[5] Gabriel SE, Helmann JD. Contributions of Zur-controlled ribosomal proteins to growth under zinc starvation conditions. J Bacteriol 2009;191:6116-22.

[6] Kuczyńska-Wiśnik D, Stojowska K, Matuszewska E, Leszczyńska D, Algara MM, Augustynowicz M, et al. Lack of intracellular trehalose affects formation of Escherichia coli persister cells. Microbiology 2015;161:786-96.

[7] Obruca S, Sedlacek P, Krzyzanek V, Mravec F, Hrubanova K, Samek O, et al. Accumulation of poly(3-hydroxybutyrate) helps bacterial cells to survive freezing. PLoS One 2016;11:e0157778.

[8] Cortes MALRM, de Carvalho CCCR. Effect of carbon sources on lipid accumulation in Rhodococcus cells. Biochem Eng J 2015;94:100-5.
[9] Suarez-Mendez CA, Sousa A, Heijnen JJ, Wahl A. Fast feast/famine cycles for studying microbial physiology under dynamic conditions: a case study with Saccharomyces cerevisiae. Metabolites 2014;4:347-72.

[10] Löffler M, Simen JD, Jäger G, Schäferhoff K, Freund A, Takors R. Engineering E coli for large-scale production - strategies considering ATP expenses and transcriptional responses. Metab Eng 2016;38:73-85.

[11] Baez A, Majdalani N, Shiloach J. Production of recombinant protein by a novel oxygen-induced system in Escherichia coli. Microb Cell Fact 2014;13:50.

[12] Ask M, Mapelli V, Höck H, Olsson L, Bettiga M. Engineering glutathione biosynthesis of Saccharomyces cerevisiae increases robustness to inhibitors in pretreated lignocellulosic materials. Microb Cell Fact 2013;12:87.

[13] Adeboye PT, Olsson L, Bettiga M. A coniferyl aldehyde dehydrogenase gene from Pseudomonas sp. strain HR199 enhances the conversion of coniferyl aldehyde by Saccharomyces cerevisiae. Bioresour Technol 2016;212:11-9.

[14] Lindahl L, Genheden S, Eriksson LA, Olsson L, Bettiga M. Sphingolipids contribute to acetic acid resistance in Zygosaccharomyces bailii. Biotechnol Bioeng 2016;113:744-53.

[15] Martani F, Marano F, Bertacchi S, Porro D, Branduardi P. The Saccharomyces cerevisiae poly(A) binding protein Pab1 as a target for eliciting stress tolerant phenotypes. Sci Rep 2015;5:18318.

[16] Knudsen JD, Carlquist M, Gorwa-Grauslund M. NADH-dependent biosensor in Saccharomyces cerevisiae: principle and validation at the single cell level. AMB Express 2014;4:81.

[17] Licznerska K, Nejman-Faleńczyk B, Bloch S, Dydecka A, Topka G, Gąsior T, et al Oxidative stress in shiga toxin production by enterohemorrhagic escherichia coli. Oxid Med Cell Longev 2016;2016:3578368.

[18] Kaloriti D, Jacobsen M, Yin Z, Patterson M, Tillmann A, Smith DA, et al Mechanisms underlying the exquisite sensitivity of Candida albicans to combinatorial cationic and oxidative stress that enhances the potent fungicidal activity of phagocytes. MBio 2014;5:e01334-01314.

[19] Jain S, Wheeler JR, Walters RW, Agrawal A, Barsic A, Parker R. ATPasemodulated stress granules contain a diverse proteome and substructure. Cell 2016;164:487-98.

[20] Yokoyama Y, Ohtaki A, Jantan I, Yohda M, Nakamoto H. Goniothalamin enhances the ATPase activity of the molecular chaperone Hsp90 but inhibits its chaperone activity. J Biochem 2015;157:161-8. 